\title{
Notas sobre Sistemas Electorales Comparados, con Especial Referencia a la Elección de Senadores Nacionales en Argentina
}

\author{
Notas sobre Sistemas Eleitorais Comparados, com Especial Referência à \\ Eleição de Senadores Nacionais na Argentina
}

Nicolás Egües

Resumen: El presente trabajo pretende analizar la importancia de los sistemas electorales para el moderno Estado de Derecho. Para tal fin, se ha centrado el análisis en el sistema de elección de los Senadores Nacionales en la Constitución Argentina y se ha comparado con los sistemas utilizados en otros estados con notas estructurales similares, concluyendo que la solución electoral que se adopte en cada caso, incide no sólo en la conformación y funcionamiento de un órgano de gobierno, sino también en otros aspectos centrales del Estado como son los niveles de representación y la participación de las Provincias en el gobierno Federal.

Palabras claves: Sistemas electorales. Representación política. Método comparado. Senadores Nacionales. Federalismo.

Resumo: Este artigo tem como objetivo analisar a importância dos sistemas eleitorais para o Estado de Direito moderno. Para o efeito, a análise centrou-se no sistema de eleição dos senadores nacionais na Constituição argentina e foi comparada com os sistemas utilizados em outros estados com notas estruturais semelhantes, concluindo que a solução eleitoral adotada em cada caso, afeta não só a formação e o funcionamento de um órgão de governo, mas também em outros aspectos centrais do Estado, como os níveis de representação e a participação das Províncias no governo federal.
Palavras-chaves: Sistemas eleitorais. Representação política. Método comparativo. Senadores nacionais. Federalismo.

Summary: This paper aims to analyze the importance of electoral systems for the modern rule of law. To that end, the analysis has focused on the system of election of National Senators in the Argentine Constitution and has been compared with the systems used in other states with similar structural notes, concluding that the electoral solution adopted in each case, affects not only the formation and functioning of a governing body, but also in other central aspects of the State such as the levels of representation and the participation of the Provinces in the Federal government.

Keywords: Electoral systems. Political representation. Comparative method. Senators. Federalism.

Artigo recebido em 30 ago. 2017 e aprovado em 9 out. 2017. 


\section{La Importancia de los Sistemas Electorales}

Antes de ingresar al aspecto central de este trabajo, entiendo corresponde efectuar algunas aclaraciones previas.

La primera de ellas se encuentra vinculada con la naturaleza, importancia y características propias de los sistemas electorales, y la segunda relacionada con el método comparativo.

Ha sostenido desde antiguo Alberdi en nuestro ámbito, que "Todo el éxito de los sistemas republicanos en países como los nuestros depende del sistema electoral"(ALBERDI, 1915, p. 153) y que "El sistema electoral es la llave del gobierno representativo"(ALBERDI, 1928, p. 103).

Lo expuesto nos da una idea sobre la centralidad del tema electoral en cualquier estado moderno de derecho, desde que es el mecanismo que permite la concreción de postulados como la soberanía popular y la representación política entendida como "un mecanismo político institucional destinado a: resolver la imposibilidad fáctica de que el pueblo se gobierne a sí mismo. (En este sentido no es un sistema de autogobierno, no supone el ejercicio directo del poder sino de delegación del poder); vincula política y jurídicamente al pueblo y al gobierno; controlar y limitar el ejercicio del poder; posibilitar la participación del electorado, a través de los representantes, en la formación de las decisiones y en el control y ejecución de las mismas; imputar la acción de los gobernantes a los gobernados; conciliar ideas e intereses de orden individual, sectorial y regional, en el marco municipal, provincial y nacional, según corresponda, para la formación de las decisiones y el control de su ejecución (GIUNTA; CUETO, 1991).

Los sistemas electorales son tan importantes que no sólo pueden concretar un ideario político, sino que también pueden llevarlo al fracaso. Podemos hablar y pregonar sobre la soberanía del pueblo, pero si luego diseñamos un sistema donde quienes acceden a votar son sólo unos pocos y lo combinamos, por ejemplo, con un sistema mayoritario donde quien gana se queda con todos los cargos en disputa, claramente estamos creando un mecanismo de representación sesgado y que en la práctica va a resultar políticamente endeble.

Esa trascendencia se refleja también en el hecho de ser estos sistemas la arena de las batallas políticas, el ámbito previo y necesario para 
que las doctrinas políticas se transformen en concreciones, al menos en un Estado de derecho ${ }^{1}$.

De nada sirve el aspecto teórico, si no se traduce en acción política y lo cierto es que la mejor manera de concretar esas acciones -aunque, claro está, no la única-, es haciéndolo desde el Estado o al menos en la disputa para acceder a los cargos electivos.

Asiste razón en este sentido a Dickinson (1981, p. 13), en cuanto a que "los detalles de la acción política son mucho más conocidos que los del debate ideológico" 2 y en tal entendimiento, resulta de especial interés para el Derecho Constitucional el estudio de los mecanismos que permiten traducir ese debate ideológico en acción, mediante el acceso a la maquinaria estatal.

No desconocemos el carácter instrumental de los sistemas electorales, pero justamente por ser herramientas tienen la primordial misión de

${ }^{1}$ Debe tenerse en cuenta que en el plano del saber político se pueden identificar al menos cuatro niveles. El primer nivel se "sitúa en el ámbito de las reflexiones sistemáticas en torno a lo político, ya sea para reconocer, describir e interpretar los fenómenos políticos, ya para profundizar sobre su esencia y la mejor forma posible de organización a que pueden aspirar los hombres. Es éste el nivel propio de la teoría política... Un segundo nivel refiere aquellas formas del pensamiento que, con menor preocupación por lo sistemático, ponen el acento en lo programático, en un proyecto por realizar en una comunidad dada. Este segundo nivel no excluye el anterior; antes bien, lo involucra como necesario antecedente en tanto el contenido programático se sostiene en apreciaciones teóricas sobre la realidad política... Quedan comprendidas en este nivel las doctrinas políticas....Un escalón más abajo, en lo que a relevancia cognoscitiva se refiere, se ubican aquellas formas de pensamiento involucradas de manera cotidiana con el momento polémico de la actividad política, con la captación de voluntades en la lucha por la obtención del poder. En este nivel, las formas más elaboradas del pensamiento se presentan de manera elemental, sin preocupación alguna por la coherencia, apuntando a conmover, encender pasiones, despertar adhesiones inmediatas. Sartori ubica aquí a las ideologías...En el último nivel, aquel más alejado de la reflexión sistemática, fuertemente involucrado con las opiniones de conjunto y con las pasiones que la actividad política pone en movimiento, se ubican los mitos, símbolos e imágenes..." Egües, Carlos A., "Objeto y método en historia de las ideas políticas", en Investigaciones y Ensayos, enero-diciembre de 1.999, Bs. As., Academia Nacional de la Historia, 1.999, p. 203.

${ }^{2}$ Partiendo de esa premisa, el autor propone en su obra, en premisa que puede extenderse el estudio de cualquier realidad constitucional, "vincular las ideas políticas con la acción política combinar lo que los hombres escribieron y dijeron acerca de la naturaleza del hombre, de la sociedad y del gobierno civil, teniendo en cuenta lo que ellos hicieron en la práctica.." ello en el convencimiento de que "el debate ideológico refleja y confronta las realidades política y social [...]".

Resenha Eleitoral (Florianópolis), v. 21, n. 1, p. 143-158, nov. 2017 
servir como nexos entre el debate sobre cómo debe organizarse el Estado y su organización concreta.

Volviendo a la representación política, la historia del Estado moderno nos da la pauta de cómo este principio se ha ido desarrollando de la mano con la implementación de diversas soluciones electorales, que han permitido su desarrollo hasta nuestros días. Se ha dicho en este sentido que (EGÜES, 2016, p. 49):

El nuevo principio de legitimidad que la teoría representativa aportó a los modernos Estados no habría superado el terreno de la formulación teórica si no se hubiera encontrado un cauce práctico, aunque complejo, que convirtiera las ideas en realidades. Las elecciones han existido a lo largo de la historia como un componente frecuente de las más diversas realidades políticas. Pero en los modernos Estados de Derecho han adquirido una importancia central, superando la condición de una técnica de designación, para integrarse en un fenómeno más sofisticado, denominado sistema electoral, que excede en mucho ese papel primario de selección de funcionarios, precisamente por la función legitimante que procuran a la organización institucional del poder.

Sería muy largo detenerse en cada una de las conquistas electorales y cómo éstas se han traducido en mejoras al concepto y práctica de la representación, pero basta como ejemplo la revolución democrática que importó para nuestra sociedad la implementación de una solución electoral integral como fue la Ley Sáenz Peña ${ }^{3}$.

En este punto, y sin intención de detenernos en aspectos históricos, cabe recordar que todo sistema electoral, o si se quiere una noción más amplia aún, toda solución electoral, se define sobre la respuesta a las preguntas de quién vota, a quién se vota, cómo se vota, cuánto se vota y dónde se vota, tal como señala Egües (2016).

$\mathrm{Y}$ en este sentido, considero que durante gran parte del Siglo XX la búsqueda de soluciones electorales se centró fundamentalmente en la ampliación de la base electoral, es decir, se detuvo sobre todo en intentar responder de manera satisfactoria la pregunta de quién vota.

\footnotetext{
${ }^{3}$ Sobre el particular puede verse Honorio A. Díaz, Ley Sáenz Peña: pro y contra, Bs. As., Centro Editor de América Latina, 1983.
} 
Hace ya muchos años Tocqueville (1955 apud HUNTINGTON, 1972, p. 16) advertía que "Entre las leyes que rigen a las sociedades humanas hay una que parece mucho más precisa y clara que todas las demás. Si los hombres quieren seguir siendo civilizados o llegar a serlo, el arte de asociarse debe crecer y perfeccionarse en la misma proporción en que aumenta la igualdad de condiciones.".

Creo que es necesario detenerse en esta cita. Al comentarla, Huntington (1972) afirma que en América Latina la igualdad en la participación política evoluciona con mayor rapidez que el arte de asociarse.

La búsqueda de la igualdad en materia electoral concentró los mayores esfuerzos, alcanzando con su logro sólo una pequeña porción de lo que implica una verdadera república democrática representativa.

Volviendo a Tocqueville (1955 apud HUNTINGTON, 1972, p. 16) y a esa suerte de confrontación que plantea entre el acceso al sufragio y lo que denomina el arte de asociarse, los desafíos actuales giran en torno a este segundo elemento. Concretado el libre acceso al sufragio de la mayor cantidad de sujetos posibles dentro de un Estado, queda por centrar los esfuerzos en los demás aspectos propios de un sistema electoral, para lograr una mejor calidad de representación y en consecuencia pulir el arte de asociarse o, en otras palabras, la relación de los individuos entre sí y de estos con el Estado.

Aparecen entonces en juego otros aspectos y pretendo detenerme mayormente en el cómo se vota y a quiénes se vota, circunscribiéndolo sólo a la elección de Senadores Nacionales.

\section{E1 Método Comparado}

El segundo aspecto a analizar antes de ingresar en el tema propuesto, tiene que ver con el estudio comparado, que resulta algo novedoso en el ámbito universitario argentino.

Se ha dicho que el "Derecho Constitucional Comparado", como lo indica su nombre, se dirige (DI RUFFIA, 2006, p. 79):

a través del llamado método comparativo, a cotejar entre sí las normas e instituciones consagradas en los diversos ordenamientos estatales, tanto del presente como del pasado, con el propósito de poner en evidencia, además de las características más significativas, sus no- 
tas similares o diferenciales, y alcanzar por esta vía la determinación posterior de principios y reglas que encuentren una efectiva aplicación en tales ordenamientos.

Con razón indican Pegoraro y Rinella (2016, p. 6) en la introducción a su obra "Derecho Constitucional Comparado", que el objeto de la publicación es su difusión mayormente en América, por cuanto se trata de un ámbito donde el Derecho Comparado en general y el Derecho Constitucional Comparado en particular, no han tenido demasiado desarrollo ${ }^{4}$.

Esta materia que constituye parte de los planes de estudio en las Universidades Europeas y que encuentra allí a sus más importantes exponentes, no ha tenido demasiado predicamento en nuestra región, en la que existen mayormente estudios dispersos sobre algunos temas particulares ${ }^{5}$.

Lo cierto es que la perspectiva comparada, enriquece el abordaje de las cuestiones institucionales porque nos permite conocer cómo se han solucionado en otros sistemas problemas similares.

Así, más allá de los fines específicos de la comparación que tienen que ver con la sistematización y organización de los conocimientos adquiridos sobre los regímenes constitucionales de diferentes Estados, existen otros fines si se quiere subsidiarios, que resultan sumamente útiles.

En este sentido, se ha afirmado que el Derecho Constitucional Comparado (PEGORARO; RINELLA, 2016, p. 331):

\footnotetext{
${ }^{4}$ Señalan los autores: "Este libro va dirigido específicamente al público ibérico y latinoamericano, donde en las universidades no se practica mucho (o no del todo) el derecho comparado (y, en particular, el Derecho constitucional comparado). Ello -espero- justifica la insistencia sobre algunos puntos, que, en otros países europeos, son consolidados: por ejemplo, la distinción entre derecho comparado y derecho extranjero, la historia de la materia, o las diferencias entre las aportaciones comparatistas de formación en derecho civil y en derecho constitucional [...]."

${ }^{5}$ Siguiendo a Biscaretti di Ruffia, puede señalarse como un estudio señero en la materia en nuestro ámbito, la obra de Segundo Linares Quintana, Tratado de la Ciencia del Derecho Constitucional argentino y comprado, Bs. As., Editorial Alfa, 1953, 9 volúmenes. Fuera del ámbito nacional y ampliando el espectro a toda América, el mismo autor refiere distintas publicaciones del Instituto de Investigaciones Jurídicas de la Universidad Nacional Autónoma de México, entre ellas: autores varios, Los sistemas federales del continente americano; Jorge Carpizo, El federalismo latinoamericano; varios autores, La evolución de la organización político constitucional de América Latina, 2 vols.; entre otras. Di Ruffia, op. cit., p. 106.
} 
es útil para los estudiosos del derecho interno en el análisis de sus ordenamientos y en la tarea correspondiente de verificación. En general, los constituyentes, los legisladores, la diplomacia y los jueces de varios países (especialmente los supremos o constitucionales) tienen presente aquello que sucede fuera de sus fronteras. Entre las llamadas funciones prácticas de la comparación existe precisamente aquella de proporcionar al legislador y a los jueces el material comparativo comentado, ordenado, clasificado, para que puedan utilizarlo con pleno conocimiento o lo que es lo mismo el estudio del derecho propio a través de la comparación ${ }^{6}$.

En materia electoral, considerando que los sistemas electorales son "estructuras complejas compuestas por una gran cantidad de elementos diferentes, los cuales pueden ser combinados casi de cualquier modo" (NOHLEN, 1995 apud EGÜES, 2016, p. 49), advertimos muchas veces la necesidad de recurrir a los modelos extranjeros para conocer de qué manera se han combinado esos elementos y cuáles han sido los resultados obtenidos, para de este modo comprender los sistemas propios y las consecuencias posibles en caso de ser modificados.

Sin ser éste un estudio estrictamente comparado, pretende al menos llamar la atención sobre algunas prácticas foráneas, para iniciar al menos el camino comparativo en los sistemas electorales americanos.

\section{El Sistema de Elección de Senadores Nacionales en la Constitución Argentina}

Desde la perspectiva reseñada en los puntos anteriores, propongo analizar rápidamente el sistema de elección de Senadores Nacionales de conformidad con las modificaciones introducidas en la reforma de 1994,

\footnotetext{
${ }^{6}$ Señala Di Ruffia entre los fines de la comparación "En primer término...la satisfacción de meras exigencias de orden cultural que se valorizan especialmente en cuanto a las posibilidades de alcanzar los lineamientos dogmáticos de la teoría general del derecho de una manera completa y satisfactoria...Existe otra finalidad que se considera de gran relieve también para aquel que pretenda permanecer en el más estricto ámbito de su propio país. La investigación comparativa, en efecto, conduce con frecuencia a una mejor interpretación y valoración de las instituciones jurídicas del ordenamiento nacional, si se tiene en cuenta que el cotejo sistemático con los ordenamientos extranjeros, especialmente si provienen de la misma cepa genealógica, podrá facilitar, en no pocas ocasiones, la identificación de principios que hasta entonces habían permanecido latentes y casi ocultos a los comentadores analíticos del derecho positivo del propio Estado [...]" Di Ruffia, op. cit., p. 80.
}

Resenha Eleitoral (Florianópolis), v. 21, n. 1, p. 143-158, nov. 2017 
puesto que allí se conjugan lo representativo, lo republicano y lo federal y se advierte cómo todos estos elementos dependen de la solución electoral adoptada.

Podemos ver aquí la influencia que ejerce el sistema electoral en el diseño institucional y cómo los elementos centrales del mismo pueden depender de la solución electoral que se adopte.

Interesa entonces determinar respecto de la reforma a la integración y elección de la Cámara de Senadores, cuál fue el análisis que se hizo de la configuración anterior, cuál fue el fundamento del cambio propuesto, cómo se plasmaron estos objetivos en la norma y finalmente qué ha pasado en la práctica.

La composición anterior al 94 del Senado nos muestra una Cámara integrada por dos Senadores elegidos por la legislatura de cada Provincia, que duraban nueve años en su cargo con renovación por tercio cada tres años ${ }^{7}$. Esta composición tiene por objeto lograr una efectiva e igualitaria participación de las Provincias en el gobierno central, prescindiendo de la población de cada una de ellas ${ }^{8}$.

${ }^{7}$ El diseño de la Constitución de 1853 se adoptó siguiendo el criterio de Alberdi, para quien "La ejecución del sistema mixto que proponemos será realizable por la división del cuerpo legislativo general en dos cámaras: una destinada a representar las provincias en su soberanía local, debiendo su elección, en segundo grado, a las legislaturas provinciales, que deben ser conservadas; y otra que, debiendo su elección al pueblo de toda la república, represente a éste, sin consideración a localidades, y como si todas las provincias formasen un solo Estado argentino. En la primera cámara serán iguales las provincias, teniendo cada una, igual número de representantes en la legislatura general; en la segunda estarán representadas según el censo de la población, y naturalmente serán desiguales... Así tendremos un congreso general, formado de dos cámaras, que será el eco de las provincias y el eco de la nación: congreso federativo y nacional a la vez, cuyas leyes serán la obra combinada de cada provincia en particular y de todas en general.” Juan Bautista Alberdi, Bases y puntos de partidas para la organización política de la República Argentina, Buenos Aires, Losada, 2003, p. 150.

${ }^{8}$ Se ha afirmado en este sentido, antes de la reforma de 1994, que "El Congreso expresa la forma representativa federal de gobierno a través de la elección directa de los diputados, que representan proporcionalmente al pueblo de la Nación, y de la elección indirecta de los Senadores, que representan a los Estados de la federación..." Humberto Quiroga Lavié, Derecho Constitucional, Bs. As., Depalma, 1987, p. 758. Completa la idea el autor afirmando que “[...] El Senado es una Cámara de reflexión, que actúa como filtro temperante del movimiento social, haciéndolo cauto y moderado. Diputados es la expresión inmediata y directa de la voluntad popular; el Senado representa sólo indirectamente al pueblo de las provincias, pero en forma directa a éstas. Diputados basa su representación en la pro- 
Todos esos aspectos fueron modificados ${ }^{9}$.

Se criticaba al momento de la reforma la duración del mandato y, sobre todo, su forma de elección. Se ha dicho en este sentido que (UBERTONE, 1999, p. 306-307):

En los años recientes hemos visto una significativa cantidad de casos de elecciones de senadores nacionales, discutibles en cuanto a la regularidad de los procedimientos y de las decisiones adoptadas. Ha habido conflictos institucionales dentro de legislaturas provinciales y entre legislaturas provinciales y el Senado nacional, y han quedado flotando algunos cuestionamientos sobre la legitimidad de algunos de los senadores incorporados al cuerpo.

Esta postura tuvo eco en el seno de la Convención Constituyente, Resultan ilustrativas en este sentido las palabras del Convencional Paixao quien afirmaba (PAIXAO, 1994, Convención Nacional Constituyente):

La configuración del órgano prevista por la constitución vigente está dada por el voto de las legislaturas provinciales y puede dar como resultado -hecho que ha sido verificado- que una fuerza política, como consecuencia de su predominio en distintos distritos, tenga una representación en el Senado, que no guarde proporción con la real relación de fuerzas del panorama político nacional. Esto puede ser consecuencia del entrecruzamiento de dos componentes que presenta actualmente el modo de integración del Senado: la elección indirecta y la excesiva duración del mandato de los senadores. A esto se

porcionalidad de la población; el Senado lo hace en la igualdad federal [...]". Humberto Quiroga Lavié, op. cit., p. 761.

9 Señala Ubertone en acertada síntesis: "el perfil del Senado nacional, en lo que se refiere a su composición, es ahora sustancialmente diferente. Veamos: a) habrá 3 senadores por provincia y 3 por la ciudad de Buenos Aires (en lugar de 2); b) los senadores serán elegidos directamente por el pueblo (en lugar de serlo de manera indirecta); c) los senadores durarán 6 años en su mandato (en lugar de 9); d) el Senado se renovará parcialmente cada 2 años (en lugar de cada 3); e) todos los senadores de cada distrito electoral serán elegidos simultáneamente; f) las bancas de cada distrito electoral se distribuirán entre los dos partidos políticos que obtengan mayor cantidad de votos en la correspondiente elección (dos para el más votado y una para el segundo)" Ubertone, Fermín Pedro, "La reforma del Senado" en Ekmekdjian, Miguel Ángel y Ferreyra, Raúl Gustavo (coordinadores), La reforma constitucional de 1994 y su influencia en el sistema republicano y democrático, Bs.As., Depalma, 1999, p. 306-307.

Resenha Eleitoral (Florianópolis), v. 21, n. 1, p. 143-158, nov. 2017 
le agrega una tercera, que es la imprevisión constitucional -lógica en el momento de la sanción de la actual Carta Magna- de representación de las minorías políticas provinciales en el Senado de la Nación.

Son estas notas, que se consideraban disvaliosas las que se pretendían subsanar con la reforma. Para ello se modificó la composición de la Cámara, la duración de los mandatos y la forma de elección de los Senadores, con la idea de lograr una mayor "democratización” del órgano y su consecuente fortalecimiento.

De este modo se disminuyó la duración de los mandatos - pasando a 6 años, con renovación por tercios cada dos-, se agregó un senador más por Provincia y se modificó el sistema de elección, pasando a una elección directa por el pueblo, asignando dos bancas al "partido político" que obtenga el mayor número de votos y una al que le siga.

En definitiva,(Cueto, 1995, p. 538):

la democratización de la Cámara, su fortalecimiento en el régimen de poder y el respeto por la calidad del órgano representativo de las provincias, fueron los objetivos explícitos y oficialmente reconocidos para el diseño. La reforma -se decía en la Convención- otorgará mayor garantía de pluralismo político, mayor certeza en la mediación entre el pueblo y sus representantes y la seguridad de que no se sentarán en las bancas personas no vinculadas con las provincias. Otorgará un alto perfil institucional y esto incrementará el poder del Congreso.

Advertimos en una primera impresión una colisión entre las ideas proclamadas como objetivo general del proceso constituyente y aquéllas que se invocaron al momento de introducir esta reforma puntual.

Es que no puede soslayarse que la reforma introducida privilegia la "democratización" o si se quiere una mayor "representatividad" en desmedro de la problemática federal, siendo esta última una de las ideas fuerza que impulsaron el proceso reformador y justamente en el órgano que está destinado a garantizar la participación de las Provincias en el gobierno central.

Encontramos aquí un claro ejemplo de cómo el rediseño del sistema electoral ha modificado directamente no sólo la composición, sino también el funcionamiento y la representación del órgano. 
Lo expuesto queda en evidencia ni bien se advierte que la representación de las Provincias en el Senado ahora replica las disputas políticas provinciales trasladándolas a la Cámara Alta, debilitando de este modo la defensa de los intereses provinciales.

Ahora los conflictos internos de la Provincia tienen eco en la Cámara, desde que uno o dos de los senadores, necesariamente integra un partido opositor al del gobernador de turno y difícilmente bregue por los intereses de su gobierno en el Senado. Esto no sólo debilita el orden federal, sino que también debilita al propio Senado.

Debe recordarse en este aspecto que nuestro modelo histórico tomaba el diseño norteamericano, que adoptó un sistema que privilegiaba la defensa de los intereses de los Estados en el Congreso Federal y obviamente, para lograrlo, debió resignar otros objetivos también deseables y válidos pero no siempre compatibles ${ }^{10}$.

A ello se agrega la consagración de que las bancas corresponden a los partidos políticos, en abierta contradicción con la conformación histórica de la Cámara, también en desmedro del sistema federal ${ }^{11}$.

${ }^{10}$ Hemos afirmado sobre el particular que "existe otro elemento que caracterizará al sistema de división de poderes norteamericano y que entendemos, resulta clave para comprender la influencia del modelo norteamericano en la Constitución argentina de 1.853.Me refiero a la especial composición social de los Estados Unidos, que ha sido caracterizada con acierto como una "sociedad homogénea en todas sus partes". Homogeneidad fundada en un fuerte igualitarismo - con las limitaciones propias de la época- y que contrasta con la composición estamental europea que sirvió de base y fuente de inspiración para los modelos propuestos por Locke y Montesquieu... La referida homogeneidad fundamentará una nueva base de representación en las Cámaras del Congreso, que no será social -puesto que no era necesaria- sino política, persiguiendo la representación de los diversos núcleos de poder propios del sistema federal consagrado en la Constitución del 87. Será entonces la puja entre los diversos Estados integrantes de la Federación la que forjará el nuevo perfil del órgano legislativo en el sistema norteamericano...Ese acuerdo necesario para compatibilizar los intereses de los diversos Estados se materializará justamente en la Constitución, que aparece no sólo como un necesario instrumento de gobierno, sino también como un verdadero pacto de unión entre los diversos estados que integran una federación.” Nicolás Egües, "Introducción al estudio del Congreso a la luz de la doctrina de la separación de poderes", publicado en suplemento Constitucional de "El Derecho" № 13.692, de fecha 19 de marzo de 2015.

${ }^{11}$ Se afirmó en el seno de la Convención que "el tema de la elección directa de senador afecta al federalismo. Se convierte al Senado en otra Cámara de Diputados. El Senado hasta ahora representaba a los gobiernos provinciales. ¿Qué va a pasar cuando los senadores dejen de sentirse atados a los gobiernos para responder directamente a los partidos?” Luis 
Los defensores de la reforma hicieron hincapié, en general, en que las modificaciones no alteraban al federalismo desde que las Provincias seguían representadas en un pie de igualdad.

Para esta postura, "lo único esencialmente federal es el principio de igualdad entre los estados provinciales. Todo lo demás puede ser modificado."

Es el convencional Saravia Toledo quien mejor define esta posición al señalar que (TOLEDO, 1994 apud CUETO, 1995, p. 552):

El Senado de la Nación no ha sido la garantía federal como fue pensado en 1853. El modo de elección de los senadores ha tenido mucho que ver. La mayoría de ellos han representado más que a los estados provinciales, a los partidos políticos que dominaban circunstancialmente las legislaturas.

Las contradicciones resultan evidentes. Si se pretende aislar al Senado de las disputas e intereses partidocráticos, no debería haberse reservado en forma expresa la titularidad de las bancas a los partidos, haciendo que en materia constitucional, el único caso de competencia exclusiva de los partidos para la postulación de candidatos sea en la elección de Senadores, exclusividad que no detentan para el resto de las elecciones, reitero, en el texto constitucional.

Pero también es contradictoria esta postura con la pretensión de mayor representatividad. Antes se criticaba la limitación que importaba la elección por parte de la legislatura, pero lejos de abrir el juego de la representación, se circunscribe todo a los partidos políticos.

Hace tiempo sostengo que una herramienta útil para restaurar el vínculo entre electores y elegidos, es el de las candidaturas independientes, no como negación de los partidos, sino como un estímulo para promover su apertura hacia la ciudadanía. Y lo cierto es que en este entendimiento, la elección directa de senadores, en un esquema de dos y uno, era una notable oportunidad para propiciar candidaturas de esta naturaleza, fuera de las estructuras partidarias.

Enseña Pérez Guihlou (1994, p. 27), comentando la reforma que:

Segundo Varese, Diario de sesiones, Convención Nacional Constituyente, $3^{\circ}$ Sesión Ordinaria, $18^{\circ}$ reunión, julio de 1994. Citado por Cueto, op. cit., p. 544. 
Los senadores representaban a las provincias como persona jurídico-política y eran movidos a actuar celosamente en sus responsabilidades con relación a sus respectivas provincias mandantes. Ahora se ha desplazado la representación a la partidocracia, que tiende a responder a los importantes intereses de los partidos que reciben sus consignas desde Buenos Aires más que desde el orden local.

Coincidimos con aquellos que afirman que la esencia del sistema federal de gobierno no sólo requiere preservar igual número de votos para todas las provincias sino también una forma de elección que resguarde el principio de que los senadores son representantes de las provincias como persona jurídico-política ${ }^{12}$.

\section{Algunas Soluciones en los Sistemas Comparados}

$\mathrm{Al}$ margen de las consideraciones vertidas hasta aquí, que no son compartidas por toda la doctrina ${ }^{13}$ (UBERTONE, 1999), entendemos que el ejemplo seleccionado sirve para ilustrar la centralidad del tema electoral.

$\mathrm{Y}$ a los fines de completar el análisis, resulta interesante verificar qué solución han adoptado otras Constituciones en el ámbito comparado.

En este sentido, la Constitución de los Estados Unidos, propone una solución similar a la que contenía nuestra Constitución histórica, de allí que hemos afirmado que este fue el modelo tomado por Alberdi y volcado en el texto de 1853. Puntualmente dispone la Sección Tercera apartado primero que "El Senado de los EE.UU. se compondrá de dos Senadores por cada Estado, elegidos por seis años por la legislatura del mismo, y cada Senador dispondrá de un voto.”

${ }^{12}$ Ver sobre el particular Cueto, op. cit.

13 Señala Ubertone entre otros aspectos positivos de la reforma que "la reforma constitucional garantiza a los partidos de oposición una apreciable cantidad de bancas en el Senado. Esto asegura o amplía el pluralismo político en ese cuerpo, y, como consideramos al pluralismo político como uno de los elementos que caracterizan a la democracia constitucional, valoramos como sumamente positiva esta innovación...”. También ha dicho que "Se suele argumentar que la división de las bancas senatoriales entre dos partidos afecta la representación de las provincias, por quitarle unidad. Tengamos claro que tal unidad de representación no existe desde que cada senador tiene un voto, $y$, aun siendo del mismo partido, puede votar uno en favor y otro en contra de un proyecto, como a veces ha sucedido. Además, el sistema permitía que las dos bancas por una provincia fueran ocupadas por senadores de distintos partidos, lo que ha sido frecuente en los años recientes; mayor probabilidad, por ende, de votos contrapuestos." Ubertone, op. cit., pág. 311.

Resenha Eleitoral (Florianópolis), v. 21, n. 1, p. 143-158, nov. 2017 
Distinta es la solución propiciada en otros Estados americanos con esquemas políticos similares -sistema federal, presidencial y con órganos legislativos bicamerales-, como es el caso de Brasil y México.

En el caso de Brasil, por ejemplo, dispone el art. 46 de la Constitución que "El Senado Federal se compone de representantes de los Estados y del Distrito Federal, elegidos según el sistema mayoritario.”

La Constitución de los Estados Unidos Mexicanos, por su parte, dispone en su artículo 56 dispone que "Para integrar la Cámara de Senadores, en cada Estado y en el Distrito Federal se elegirán cuatro senadores, de los cuales tres serán electos según el principio de votación mayoritaria relativa y uno será asignado a la primera minoría. Para cada entidad federativa, los partidos políticos deberán registrar una lista con tres fórmulas de candidatos. La senaduría de primera minoría le será asignada a la fórmula de candidatos que encabece la lista del partido político que, por sí mismo, haya ocupado el segundo lugar en número de votos en la entidad de que se trate. La Cámara de Senadores se renovará en su totalidad, en elección directa, cada seis años."

Advertimos en estos ejemplos, la adopción de diversas fórmulas para lograr la representación de los estados locales en la Cámara de Senadores.

En algunos casos se privilegia la representación orgánica-institucional, tal el modelo norteamericano y en otros se privilegia una mayor apertura al electorado, con el riesgo de que la representación de los estado se vea atenuada o desvirtuada. Entre estos ejemplos, destaca la diferencia entre los modelos que asignan directamente la banca a los partidos -caso de Argentina y México- y de aquellos que no lo hacen de manera expresa - Brasil.

Una primera conclusión, a la luz del funcionamiento real de la Cámara de Senadores en cada uno de los Estados analizados, indica la posible inconveniencia de asignar la banca a los partidos.

\section{Conclusión}

Finalizando con el análisis propuesto, advierto que la elección de Senadores es una clara muestra de la importancia en el diseño de un sistema electoral y como éste puede repercutir en el funcionamiento del Estado en su conjunto. 
Resulta necesario remitirse a las palabras de Alberdi una y otra vez: el sistema electoral es la llave de la república, es la clave para el diseño institucional y de él dependerá de qué manera y en qué medida podamos hacer realidad la vigencia de nuestro sistema representativo, republicano y federal.

La actual composición del Senado ha debilitado al federalismo, justo en la Cámara que representa la esencia del sistema Federal. Y ello, so pretexto de una mayor apertura a la ciudadanía que es cierta sólo a medias.

Finalmente, esa división por un lado y traslación por otro de la representación, ha debilitado no sólo a la Cámara sino al Congreso en su conjunto, no obstante estar conscientes de que no es la única ni la más importante de las causas de la opacidad del Congreso Argentino.

La realidad demuestra que los representantes de las Provincias sólo siguen los mandatos partidarios, tal como se preveía, y que rara vez puede verse encolumnados a los tres senadores de una Provincia en la lucha por los intereses de ésta.

Volviendo a Alberdi (op. cit., p. 87), advertimos que su ideal no se ha concretado y que lejos estamos todavía de ser dignos de "la república, que hemos proclamado, que no podemos practicar hoy ni tampoco abandonar [...]."

No queda más consuelo que saber que "el camino es largo y hay mucho que esperar hasta llegar a su fin [...]”. (ibidem)

\section{Referencias}

ALBERDI, Juan Bautista. Bases y puntos de partida para la organización política de la República Argentina. Bs. As., La cultura Argentina, 1915, Cap. XXII.

ALBERDI, Juan Bautista. Derecho Público Provincial Argentino. Bs.As., Talleres Gráficos Argentinos de IJ Rosso, 1928.

CUETO, Walter José. El Senado por venir. In: GUILHOU; SEISDEDOS y otros, Derecho Constitucional de la Reforma de 1994. Mendoza, Martín Fierro, 1995, T. I, p. 538.

DICKINSON, H.T. . Libertad y propiedad. Ideología política británica del siglo XVIII. Bs. As., EUDEBA, 1981. 
DI RUFFIA, Paolo Biscaretti. Introducción al derecho constitucional comparado. Las "formas de Estado" y las "formas de gobierno". Las Constituciones modernas. México, Fondo de Cultura Económico, 2006.

EGÜES, Carlos A.. Representación política y sistema electoral. República y Derecho Revista Jurídica, Volumen I, MMXVI, p. 49.

GIUNTA; María Luisa; CUETO, Walter Cueto. La reforma legislativa. Una propuesta de diseño constitucional. Mendoza, Depalma, 1991, pág. 51.

GUILHOU, Dardo Pérez. Prólogo a la Constitución de la Nación Argentina. Bs. As., Depalma, 1994, p. XXVII.

HUNTINGTON, Samuel P.. El orden político en las sociedades en cambio. Bs. As., Paidós, 1972.

PAIXAO, Enrique. Diario de sesiones, Convención Nacional Constituyente, $3^{\circ}$ Sesión Ordinaria, $18^{\circ}$ reunión, jul 1994.

PEGORARO, Lucio; RINELLA, Angelo. (Coord.) Derecho Constitucional Comparado. Bs. As., Astrea, 2016, T. I, p. 6.

UBERTONE, Fermín Pedro. La reforma del Senado. In: EKMEKDJIAN, Miguel Ángel; FERREYRA, Raúl Gustavo (Coord.). La reforma constitucional de 1994 y su influencia en el sistema republicano y democrático. Bs.As., Depalma, 1999, pp. 306-307.

Nicolás Egües - Abogado. Profesor de Derecho Constitucional en la Facultad de Ciencias Políticas y Sociales de la Universidad Nacional de Cuyo. Profesor de Derecho Constitucional y de Derecho Procesal Constitucional en la Facultad de Ciencias Jurídicas y Sociales de la Universidad de Mendoza. Miembro titular y actual vicepresidente del Instituto Argentino de Estudios Constitucionales y Políticos. 